\title{
Segmenting the Etiological Agent of Schistosomiasis for High-Content Screening
}

\author{
Daniel E. Asarnow and Rahul Singh*, Member, IEEE
}

\begin{abstract}
Schistosomiasis is a parasitic disease with a global health impact second only to malaria. The World Health Organization has classified schistosomiasis as an illness for which new therapies are urgently needed. However, the causative parasite is refractory to current high-throughput drug screening due to the diversity and complexity of shape, appearance and movement-based phenotypes exhibited in response to putative drugs. Currently, there is no automated image-based approach capable of relieving this deficiency. We propose and validate an image segmentation algorithm designed to overcome the distinct challenges posed by schistosomes and macroparasites in general, including irregular shapes and sizes, dense groups of touching parasites and the unpredictable effects of drug exposure. Our approach combines a region-based distributing function with a novel edge detector derived from phase congruency and grayscale thinning by threshold superposition. The method is sufficiently rapid, robust and accurate to be used for quantitative analysis of diverse parasite phenotypes in high-throughput and high-content screening.
\end{abstract}

Index Terms-Drug discovery, grayscale morphology, high-throughput screening, image segmentation, phase congruency, schistosomiasis.

\section{INTRODUCTION}

\section{A. Disease Background}

$\mathbf{S}$ CHISTOSOMIASIS is a parasitic disease considered to have global health and socio-economic impacts second only to malaria. Although incidence of the disease in developed countries is extremely low, more than 200 million people are infected worldwide, with an additional 800 million at risk. The chronic illness is caused by infection with one of several species of trematodes, chiefly Schistosoma mansoni, Schistosoma haematobium, and Schistosoma japonicum, which are carried to humans through water contaminated with their larvae. Early on, infection is characterized by an inflammatory response to the parasites' eggs, eventually leading to fibrotic

Manuscript received December 12, 2012; accepted January 11, 2013. Date of publication February 14, 2013; date of current version May 29, 2013. This work was supported in part by the National Institutes of Health, National Institute of Allergy and Infectious Diseases (NIH-NIAID) under Grant 1R01AI089896, in part by the National Science Foundation (NSF) under Grant IIS-0644418 (CAREER), and in part by the California State University Program for Research and Education in Biotechnology (CSUPERB). Asterisk indicates corresponding author.

D. E. Asarnow is with the Department of Chemistry and Biochemistry, San Francisco State University, San Francisco, CA 94132 USA.

*R. Singh is with the Department of Computer Science, San Francisco State University, San Francisco, CA 94132 USA (e-mail: rahul@sfsu.edu).

Color versions of one or more of the figures in this paper are available online at http://ieeexplore.ieee.org.

Digital Object Identifier 10.1109/TMI.2013.2247412 granulomas that can occlude the hepatic portal vein and cause hydronephrosis (kidney swelling from urine buildup) and squamous cell bladder cancer. Other effects of schistosomiasis include diarrhea, lesions in the central nervous system and genital sores which enhance the transmission of HIV. The World Health Organization (WHO) has classified schistosomiasis as one of 17 neglected tropical diseases (NTD), a set of illnesses grouped together because they 1) are proxies for poverty, 2) affect politically disadvantaged populations, 3) do not travel out of the third world, 4) lead to discrimination, especially of women, 5) have serious, widespread health effects, 6) are neglected by research and 7) might be controlled through currently feasible means [1].

For nearly 40 years, the drug Praziquantel (PZQ) has provided what is essentially the only avenue of treatment for schistosomiasis. While PZQ has some desirable properties-it is a single-dose drug effective against all major Schistosoma species which infect humans - it also has a number of side effects, as well as a variable rate-of-cure as low as $60 \%$ and a significantly lower activity against juvenile parasites [2]. WHO considers schistosomiasis a disease for which new treatments are urgently needed [3].

\section{B. Automated Screening Against Schistosomiasis}

Modern drug discovery pipelines employ either target-based screens, using in vitro assays of individual molecules, or phenotypic screens of entire disease systems. Typically a large number of molecules are screened, since even small structural variations can lead to significant changes in activity against the target. Due to the need for large compound libraries, automation is a critical component, however phenotypic screens are often refractory to automation, requiring a human expert for handling and testing a target organism as well for analysis of phenotypic data. At the same time, phenotypic screens are inherently holistic - that is, such screens may identify drugs whose efficacy derives from unknown molecular mechanisms of action, which may be significantly more complex than those proposed in target-based assays. It is thus unsurprising that there is evidence that phenotypic assays are significantly more effective than target-based screens, and that an overemphasis on the latter may be responsible for the high attrition rate $(\sim 95 \%)$ of drug candidates experienced in the industry today [4].

\section{Role of Image Segmentation}

Recently, efforts have been initiated towards development of automated, high content phenotypic screens (henceforth abbreviated as HCS) for schistosomiasis [5], [6]. An important problem encountered in these efforts has been the consistent 
and accurate segmentation of the parasite which is critical to the measurement and quantification of their anatomical, appearance-based, and motion-based phenotypes and thus to successful automation of a screen for drug compounds.

In this paper, we propose an image segmentation algorithm for bright-field microscopy images of the juvenile schistosomula, laying the fundamental foundation of a complete computer vision system for this domain. The proposed algorithm segments the scene, obtaining a region mask which may be used to extract the individual parasites present in each frame. Multidimensional measurements can then be used to construct and analyze time series of arbitrary descriptors capturing parasite shape (e.g., geometry, curvature), parasite appearance (e.g., color, texture) and parasite motion (e.g., image difference). These measurements can form a key pillar of a larger system for automated drug discovery, which seeks to use a quantitative, holistic description of the phenotypes elicited by drug compounds as a descriptive, and eventually predictive, bridge between small molecules and disease system phenotypes.

The principal contributions of this work are as follows.

- We propose a new binary edge classifier employing phase congruency and gray-scale thinning by threshold superposition. This edge detector can accurately locate weak, perceptual features at arbitrary angles of phase without relying on an estimation of feature orientations.

- Parsimonious assignment of foreground pixels with ambiguous region membership, permitting edge-based splitting without corruption of region boundaries by noisy edges or spurious fragmentation of irregularly shaped objects.

- A segmentation algorithm which is highly accurate and sufficiently fast so as to extract detailed, quantitative descriptions of parasite appearance and behavior that can be used to develop automated, high-throughput phenotypic screens for drug screening against schistosomiasis.

To the best of our knowledge, this is the first work which looks at the problem of automatic segmentation of parasites underlying NTD from the perspective of HCS.

\section{Problem Formulation and Characteristics}

A key consideration in high-content phenotypic screening is precise measurements of the effects of a compound on the shape, appearance, and motion of parasites. Consequently, a segmentation method must not only delineate parasites from the background and from each other but also be highly accurate in doing so. Furthermore, the method must remain robust under variation in illumination and image capture conditions.

Fig. 1 illustrates the great phenotypic diversity of schistosomula under the effects of different drugs. The reader may note, among others, the highly irregular parasite shapes, as well as the strong intensity variation between parasites, internal inhomogeneities, and touching parasites. Segmentation of schistosomula in particular thus raises challenges which are distinct from the segmentation of cells, which has been an active area of research in biological imaging. Specifically, these challenges include the following.

- The parasites are all unique individuals and they cannot be cloned. They exhibit marked variation in size, shape, and

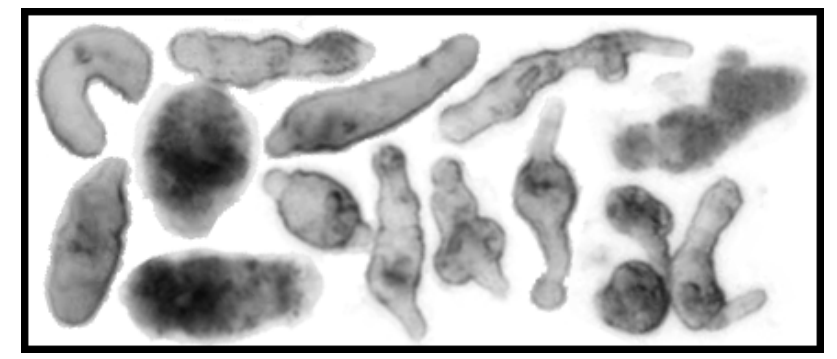

Fig. 1. Illustration of the phenotypic diversity of schistosomula, with and without drug exposure. The reader may note touching parasites and extreme differences in body shape as well as color, texture, and visibility of internal structures.

movement patterns. In addition, the elongation and contraction of the musculature which enables schistosomula to move can result in drastic alterations of proportionality, shape and orientation. These facts preclude the assumption of an a priori geometric shape model.

- Additional variation in color, texture and edge strength arises due to differences between individuals as well as their movement. For example, contractive periods (where the concentration of material is higher in the middle of the parasite body) lead to differential contrast with the image background as compared to periods of relaxation.

- The proclivity of schistosomula to touch or overlap slightly. This leads to the formation of large groups of parasites in physical contact. Touching parasites are likely to be segmented as a single object, as the edges between them may be weak or nonexistent.

- The presence of visible anatomical structures within the parasites. These structures (such as the digestive tract) create internal edges which do not correspond to parasite boundaries.

- Debris which accumulate over long periods of observation or which are inadvertently introduced during preparation of an experiment. These can complicate parasite identification and segmentation.

- Alterations in the visual appearance of the parasites due to the effects of drug exposure. We underline that the phenotypes exhibited by individual parasites may differ due to lack of genetic homogeneity.

- The rigors of high-throughput. High resolution images of large collections of parasites must be processed with rapidity. Parallelizability, numerical stability and convergence guarantees are all highly desirable.

A proposed method must successfully address each of these difficulties in addition to the requirements of general applicability and adaptability. Consistency and accuracy of segmentation are also of paramount importance to ensure that sensitive measurements of the impact of drugs may be made.

\section{PRIOR WORK}

Owing to the challenges discussed above, till date, automated methods have only just been introduced for screening drugs against helminthic diseases. The only such screening system [7] aside from our previous work [5], [6] does not employ a novel segmentation algorithm and in particular simply discards 
touching or highly irregular individuals. In contrast to parasites, segmentation and tracking of cells has been an active research area for some time, and several approaches undertaking the segmentation of touching cells have been proposed. There are also widely available tools for cell segmentation by nonspecialists, such as the CellProfiler suite which provides a visual programming environment for quantitation of images of cells [25]. In addition to cells, $C$. elegans is visually similar in some respects to vermiform macroparasites such as the Schistosomatidae, and attempts have been made to segment touching individuals. As described below, methods developed in these contexts all suffer from one or more specific limitations relevant to our problem domain.

Methods employing articulated models [8] and path searching on probabilistic shape models [9] have been used for $C$. elegans, while cells have been segmented by fitting of elliptical models [10] and statistical merging of manually constructed models [11]. However, explicit models of size, shape and/or intensity are impractical for schistosomula due to the natural variation of individuals and the extreme and unpredictable variation induced by drug exposure

A number of methods attempt to separate touching objects based on the watershed transform, including region merging [12], [13], iterative erosions for marker localization [14] and minima merging [15]. All these methods require good seed points, obtained from user interaction or alternate image channels (such as fluorescence) which are unavailable for schistosomula.

Active contours are attractive because they can adapt to arbitrary shapes, however in practice topological discontinuities prevent them from robustly segmenting touching objects. The addition of repulsive terms is one possible solution, but manual initialization of individual contours is required in many formulations [16]. Using the level set method avoids topological difficulties - the Chan-Vese [17] formulation, used e.g., in [18], provides a popular approach which has been further specialized for touching objects by combination with watersheds [19] or by taking into account mixing of object boundaries [20]. In practice, the gradient forces used are subject to the same shortcomings discussed in Section V-A, and all of [18]-[20] rely on seeds and/or boundary information obtained from fluorescent dyes.

A distinct approach to segmenting touching cells employs max-flow/min-cut followed by refinement using $\alpha$-expansions and graph coloring [21]. The graph-cut refinement takes advantage of a centrally located intensity maximum at the cell nucleus to produce high quality seeds, an effect not exhibited by schistosomes. Another set of approaches uses graph partitioning with normalized cuts [22]. The normalized cuts algorithm has two major disadvantages in the present context. First, the number of segments must be specified by the user and second, the computational complexity is cubic in the size of the image.

Diverging from other methods discussed so far, the ilastik system employs an active supervision procedure in which a user manually brushes (with a pointing device) swathes of representative images corresponding to the desired classes [23]. A random forest classifier is then trained on features from the selected regions, using a number of classical feature extractors. In the context of HCS involving diverse and unanticipated phenotypes, supervised solutions may be disadvantaged. Such methods may however be acceptable if the training is efficient with respect to human effort and if training sets that represent the diversity in the data can be created. Ilastik, along with a variety of general image processing algorithms, such as histogram-based thresholding, watershed segmentation and mathematical morphology are all available in CellProfiler. We used CellProfiler to implement certain alternate approaches to segmentation for comparison with the proposed method, which are discussed further in Section VII-A.

Expanding the principle of active contours to regions, Srinivasa et al. [24] devise an "Active Mask" algorithm in which region masks are iteratively evolved and/or discarded in order to produce a correct labeling of each pixel. A pixel weight for each mask is assigned on the basis of region-based and voting-based distributing functions. Active Mask benefits from the quality of the background weights given by the region-based distributing function, the direct evolution of region masks under the voting functions and the use of a random initialization (rather than particular seeds). It also suffers from high computational complexity, in time and space, which is exacerbated because masks can only be discarded and not introduced $a b$ initio. A large number (many more than the likely number of regions) of initial masks must be used to permit smooth convergence to region boundaries and to avoid missing objects entirely. Furthermore, the voting-based distributing functions assume an elliptical shape.

Fig. 2 presents a side-by-side comparison of schistosomula images segmented using several of the previous methods. Note that though each of these actively attempts to separate touching objects, they are unable to do so correctly in images from our problem domain. Although Active Mask is not completely successful, when the number of masks is forced to be 1 , the segmentation becomes entirely determined by the region-based distributing function. The result is superior foreground recognition, at the cost of the ability to identify separate regions via their individual masks. The inadequate performance of these methods and especially their inability to separate touching parasites indicates a need for novel techniques.

\section{MeTHOd OVERVIEW}

The proposed algorithm consists in the following major components.

- Initial segmentation. Rapid background-foreground segmentation is achieved using morphological preprocessing and a region-based distributing function (RBDF). Initial regions represent one or more objects and are individually refined using grayscale features.

- Edge detection and topological correction. Edge information extracted by the novel edge detector is used to enforce the correct topology in terms of perceptual connectivity of objects.

- Cleanup and reassembly. The refined, segmented regions are subjected to morphological cleaning and compiled into a final binary mask.

To elaborate on the above, separation of merged parasites is performed by extracting edge features from the grayscale sub-image using a novel algorithm which combines spectral 


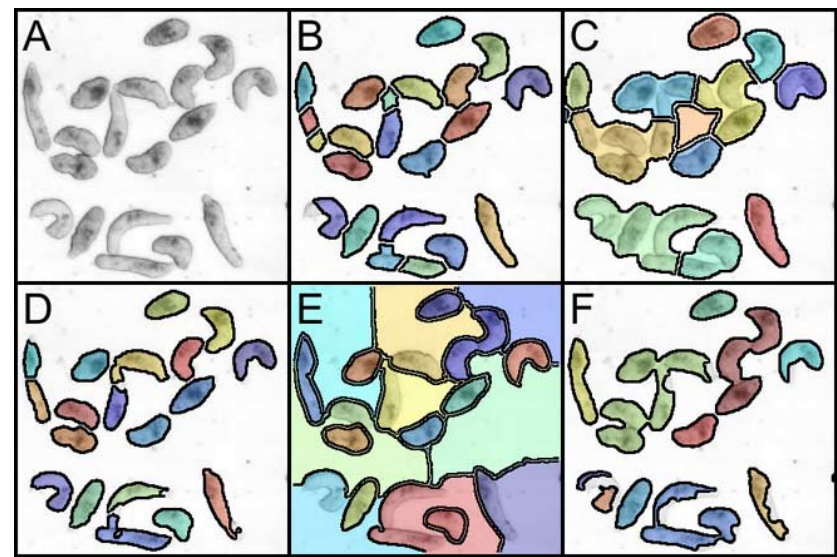

Fig. 2. Segmentation by extant methods. (A) Original subimage. (B) Otsu's method/watersheds. (C) Active Mask. (D) Ilastik/watersheds. (E) Normalized cuts. (F) Level set method. Colored overlay represents region indexes, black lines indicate region boundaries.

and morphological methods, including phase congruency and grayscale thinning by threshold superposition. Extracted edge features are used to impose a new topology which correctly reflects the boundaries and connectedness of the objects present in the scene. In particular, we use the watershed transform to derive minimal edges which reproduce the correct topology while satisfying the principle of maximum parsimony: the fewest edge points are associated with the fewest number of objects across the shortest distances possible. This permits the recognition of touching individuals, while maintaining a high degree of accuracy with respect to the precise positioning and extent of each parasite. Seed points, alternate image channels, user interaction and $a$ priori modeling are all completely avoided.

In the following subsections each step of our approach is described in detail. Fig. 3 illustrates the major stages of the algorithm as they are applied to a particular ROI exhibiting the various challenges enumerated previously.

\section{INITIAL SEgmENTATION}

To prepare an image for segmentation, uneven lighting is corrected using the top hat transform. The initial foreground-background separation is obtained by applying a global threshold to a low-pass filtered copy of the image. This approach is motivated by the region-based distributing function (RBDF) in Active Mask [26]. In addition to a low-pass filter, Active Mask employs soft-thresholding with a sigmoidal curve (the error function). The purpose is to derive continuous pixel weights for foreground membership, which saturate at pixels with high-confidence classification-essentially mimicking a phase transition across region boundaries. In the context of a global threshold, the saturation points can be used to derive intensity intervals for controlled over- or under-segmentation of the foreground. After extensive experimentation we elected to use the global threshold directly. Given the image $f$, threshold $\gamma$ and a low-pass filter $h$, the foreground mask $R$ is given by

$$
R(x)= \begin{cases}1, & (f * h)(x) \leq \gamma \\ 0, & (f * h)(x)>\gamma\end{cases}
$$

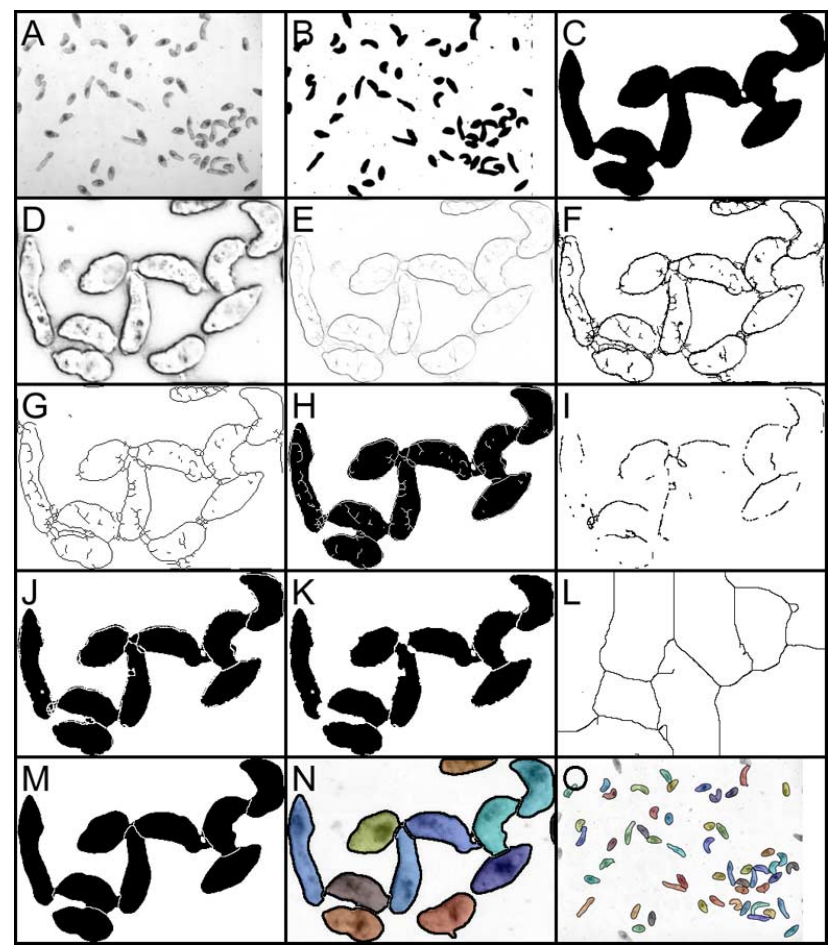

Fig. 3. Illustration of proposed method. (A) Original $1388 \times 1040$ image. (B) Foreground identification. (C) ROI containing several merged parasites. (D) Phase congruency of the ROI. (E) Thinned phase congruency. (F) Binary hysteresis edges. (G) Cleaned edges. (H) Edge-subtracted marker regions. (I) Relevant edges, which separate edge markers. (J) ROI with correct region topology. (K) Morphological cleaning to remove noise propagated from edge detection. (L) Watershed lines indicate the influence zones of the corrected regions. (M) ROI refined by parsimonious assignment of pixels to regions. (N) ROI segmentation overlay. (O) Final segmentation overlay. In this example, every parasite is correctly identified by the method, as shown in $(\mathrm{N})$ and $(\mathrm{O})$. Parts (B)-(M) inverted for print.

We use a radially symmetric Gaussian low-pass filter $h(x)=$ $e^{-|x|^{2} / a^{2}}$ with scale $a$ set to about the size of a typical edge. The low-pass filter thus serves to reduce the noise along region boundaries, while blurring heterogeneous pixels within regions which might otherwise lead to gaps in the foreground mask. The end result is a more conservative threshold with respect to the foreground. While $\gamma$ here represents a threshold delineating pixel classes, in [26] the difference between class intensities is used, and must be specified manually.

Appropriate selection of the foreground classification threshold $\gamma$ is critical to the quality of the initial segmentation. We had previously estimated $\gamma$ on the basis of an iterative search for the value producing the largest increase in the number of segmented regions, as the intensity value is decreased within the range found in the image [27]. However, this approach is applicable only when levels of noise and debris artifacts (small spots) in the image are low. A more robust value for $\gamma$ might be found using Otsu's method [28], however bias arises because Otsu's method does not consider overlap between foreground and background distributions. Nevertheless, the result is consistent and works fairly well if a bias correction factor of 0.5 is applied. However, an unbiased threshold estimator which does not require such adjustment is preferable. 


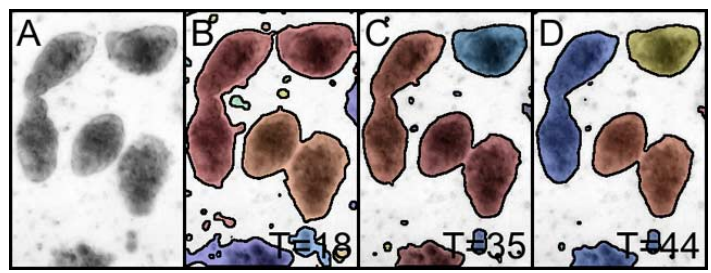

Fig. 4. Comparison of threshold selection methods. (A) Part of a parasite image. (B) iterative threshold estimation $(T=18)$. (C) Otsu's method $(T=35)$. (D) Maximum likelihood threshold estimation $(T=44)$.

TABLE I

ACCURACY OF THRESHOLd ESTIMATES FOR INITIAL SEgMENTATION

\begin{tabular}{|c|c|c|}
\hline Method & Precision & Recall \\
\hline Iterative estimate & 0.999 & 0.453 \\
\hline Otsu w/ bias correction & 0.994 & 0.779 \\
\hline Maximum likelihood & 0.971 & 0.862 \\
\hline Maximum entropy & 0.989 & 0.814 \\
\hline Minimmum error & 0.997 & 0.707 \\
\hline Minimum error (Poisson) & 0.748 & 0.920 \\
\hline
\end{tabular}

An unbiased threshold can be obtained using an expectation-maximization (EM) calculation of the threshold satisfying the maximum likelihood (ML) criterion. The EM algorithm, which accounts for overlap between the Gaussian distributions which model the histogram [29], is initialized using Otsu's method. The consideration of overlap between pixel distributions is especially important because of the large size of our images with respect to the imaged parasites leads to a large background distribution which overlaps heavily with the foreground in the intensity histogram. In addition to providing an unbiased threshold, the EM algorithm is guaranteed to converge [30]. When ML is used to determine $\gamma$ prior to application of the RBDF the result is a consistent, but slight, over-segmentation which ensures that all or nearly all of the true foreground area is captured in each image. In general, no foreground regions are missed outright during initial segmentation.

Fig. 4 presents an example RBDF result for the original iterative method, Otsu's method and the ML method. Furthermore, the ground truth data described in Section VII permits a quantitative evaluation of alternate threshold estimation methods (before any morphological post processing), which is shown in Table I. In the evaluations presented in this table, maximum entropy [29], minimum error [29] and minimum error using Poisson distributions [31] are considered in addition to Otsu's method and ML. As described further in Section VII-A, precision and recall values are calculated using all pixels in each object. In general, precision $<95 \%$ indicates an unacceptable level of true foreground missed, while recall $<85 \%$ indicates unacceptable amounts of oversegmentation.

The accuracy of the intentionally over-segmented foreground mask is improved by a selective erosion operation which preserves pixels along the image border, unless they are part of a region whose intersection with the border is smaller than a predefined limit. This prevents regions which intersect the border only tangentially from being deleted when objects truncated at the border are removed. Small regions created by spotting of the

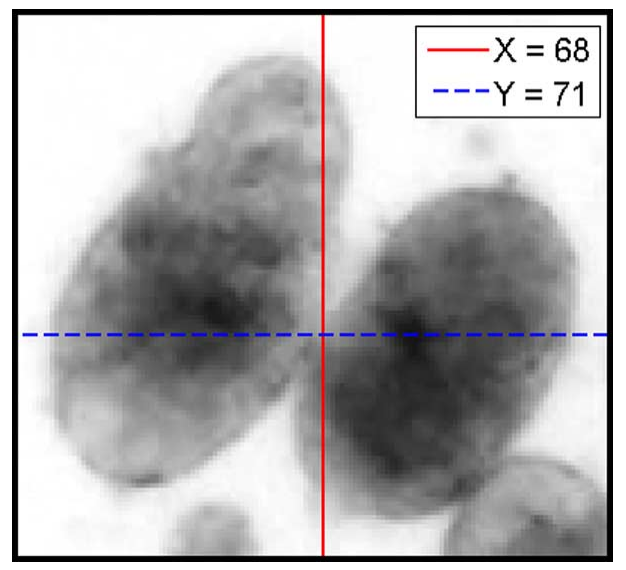

Fig. 5. Small area from a schistosome image. The dashed, horizontal line indicates the intensity cross section graphed in Fig. 6. Solid, vertical line matches that of Fig. 6. The parasites in this image have been treated with the drug Simvastatin.

media, debris in the well or decomposing pieces of the parasite tegument (outer cell layer) are removed as well.

Schistosomula often touch, forming large groups or clumps, and spuriously merged regions are common in the initial segmentation [Fig. 3(b) and (c)]. Each discrete 8-connected component in the initial segmentation (potentially representing several parasites) is taken as a region of interest (ROI) and refined individually in turn. In the following, we represent the partitioning of an image - a set of pixels $X=\left\{x_{i}\right\}$ with intensities $I_{x}$ (Boolean-valued for the binary case)-into connected components as $\prod_{i}(X)$. For each connected component $C_{i}$, the minimal bounding rectangle is computed and used to extract the corresponding grayscale and binary sub-images.

\section{Edge Detection and Topological Correction}

\section{A. Edge Detection}

The first step in the refinement process is edge detection. Traditional edge detection methods rely on the image gradient. The best known examples of such methods are the Prewitt-Sobel-Roberts family of derivative approximations and especially the Canny operator [32]. Gradient-based edge detectors applied to the schistosomula images produce edges which are insufficiently accurate to separate merged parasites without falsely splitting individual ones. Permissive thresholds are characterized by a high degree of noise and artifacting, while conservative thresholds fail to detect the weak edges which often occur between touching parasites. Furthermore, such methods suffer from a "double edge" artifact which prevents edges wide enough to have only a small gradient in the center from being identified correctly.

Our approach addresses these issues with a novel edge detector aimed at producing accurate edge contours with maximum perceptual salience. Rather than the intensity gradient, we use the phase congruency of the grayscale image. phase congruency (PC) is an approach to feature detection based on the Local Energy Model [33], which holds that perceptually salient 


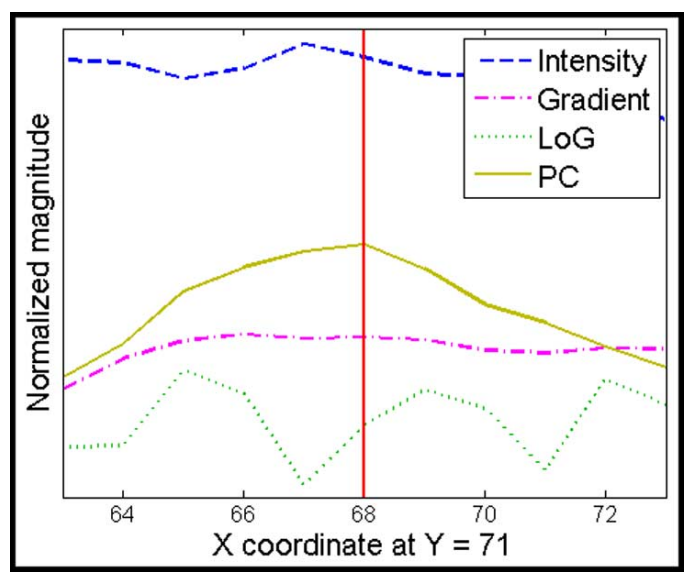

Fig. 6. Graph of intensity edge-weight profiles. The solid line indicates an intensity cross section (horizontal line in Fig. 5). Dashed, dotted and dashed/dotted lines indicate the corresponding intensity gradient, and the magnitudes of the Laplacian of Gaussian and Phase Congruency operators, respectively. Note that only the local maximum of the phase congruency curve correctly localizes the weak edge between the parasites shown in Fig. 5 .

features occur where an image's Fourier components are maximally in phase with one another.

Phase congruency has a number of qualities which are advantageous in comparison to image gradients. First, PC is a dimensionless quantity restricted to the interval [ $\left[\begin{array}{ll}0 & 1\end{array}\right]$, simplifying thresholding. Second, this notion is illumination and contrast invariant, and can detect perceptual elements which do not coincide with steps in the image gradient, and are characterized by arbitrary phases. Finally, PC is naturally multi-scale and can be implemented efficiently using fast wavelet transforms [34]. These advantages relative to spatial methods can be demonstrated by examining a cross-section of the image shown in Fig. 5. The horizontal and vertical lines in Fig. 5 indicate the cross-section in question, and the position of a perceptual feature along that cross-section, respectively. Fig. 6 graphs the intensity profile along the cross-section, as well as corresponding profiles from the gradient magnitude, the response of a Laplacian of a Gaussian filter and the phase congruency of the image. The gradient is computed using the highly accurate 7-tap FIR filter given in [35]. Also shown is a vertical line, matching that of Fig. 5, which indicates the perceptual feature of interest. While this edge is characterized by phases to which the gradient and LoG filter are insensitive, phase congruency robustly detects the edge.

Mathematically, phase congruency is defined as the ratio between the local energy, or absolute magnitude in frequency space, and the total path length of all the (complex) frequency component vectors $A_{n}$. If $F(x)$ denotes the real components of the spatial frequencies and $H(x)$ the imaginary component, then the local energy is defined by

$$
E(x)=\sqrt{F^{2}(x)+H^{2}(x)} .
$$

It is desirable to consider the diversity of the frequency components which contribute to the overall congruency; features which are characterized by many in-phase components should receive a higher weight than those with just a few. The weights are provided by a weighting function $W(x)$ of the local frequency spread $s(x)$

$$
\begin{aligned}
s(x) & =\left(\frac{1}{N}\right) \frac{\sum_{n} A_{n}(x)}{\varepsilon+A_{\max }(x)} \\
W(x) & =\frac{1}{1+e^{\beta(c-s(x))}} .
\end{aligned}
$$

The quantity $s(x)$ ranges from 0 (if a single frequency component contributes) to 1 (if all are equal). The value $\varepsilon$ is a small constant representing numerical precision, included to prevent ill-conditioned behavior in cases where all frequency components are very small.

The sigmoidal $W(x)$ is designed to penalize overly narrow frequency distributions; its parameters $\beta$ and $c$ determine the sharpness of the sigmoid transition and the cutoff below which a penalty is applied. In addition, noise is cancelled by using the smallest wavelet scale to estimate the average noise energy $T$, beyond which frequency amplitude vectors must extend in order to be considered signal. The reader is referred to [34] for a detailed description of the weighting function $W(x)$ and its parameters, as well as the noise cancellation procedure.

The full PC expression, including effects of noise and frequency spread, is given by

$$
P C(x)= \begin{cases}\frac{W(x)(E(x)-T)}{\sum_{n} A_{n}(x)+\varepsilon}, & E(x)-T>0 \\ 0, & E(x)-T \leq 0\end{cases}
$$

Calculating phase congruency requires a local, phase-preserving frequency analysis in order to fully determine each complex frequency component vector $A_{n}$. An appropriate method is the wavelet transform, using quadrature pairs of matched even and odd filters. Frequency information (energy $E$ and phase angle $\phi$ ) is then extracted by comparing responses to the symmetric filter $f$ and antisymmetric filter $h$ via

$$
\begin{aligned}
& E(x)=\sqrt{f(x)^{2}+h(x)^{2}} \\
& \phi(x)=\operatorname{atan} 2(f(x), h(x)) .
\end{aligned}
$$

Log-Gabor wavelets, characterized by a Gaussian transfer function on a logarithmically scaled frequency axis, are chosen because they are psychophysically justified [36], and because they possess zero mean at arbitrary bandwidths. The log-Gabor transfer function with center frequency $\omega_{0}$ is given by

$$
\mathcal{G}(\omega)=e^{-\ln \left(\omega / \omega_{0}\right)^{2} / 2 \ln \left(\kappa / \omega_{0}\right)^{2}} .
$$

Analysis at multiple scales is conducted by summing the responses to a bank of log-Gabor filters. This sense of scale, defined over a range by the extent of the tail of the log-Gaussian distribution in frequency space, is attractive because it derives not from the spatial extent of a feature, but from the spatial extent of its constituent frequency components.

\section{B. Ridge Detection and Binary Classification}

In general, the edge weights obtained via phase congruency (or gradient methods) are, for a given image feature, diffused over a width greater than that needed or desired for edge based region splitting [Fig. 3(d)]. In order to accurately localize edges, 


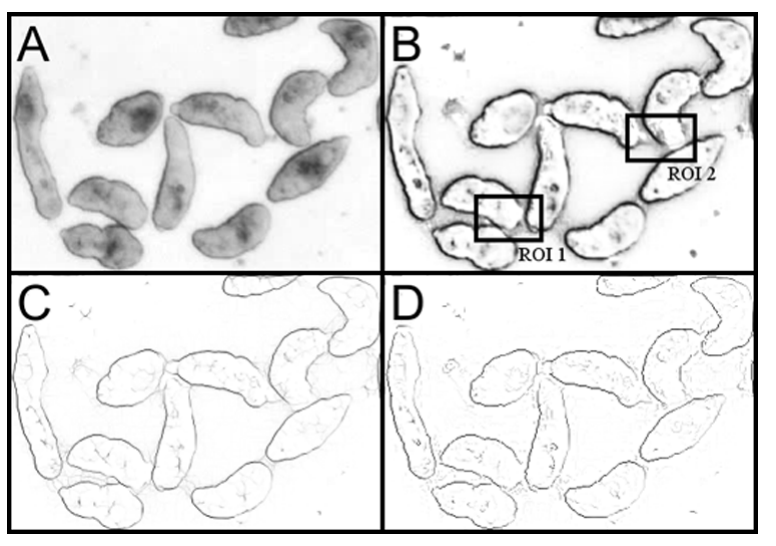

Fig. 7. Phase congruency and ridge detection. (A) Grayscale image. (B) Phase congruency. (C) Grayscale thinning. (D) Nonmaxima suppression. (B)-(D) inverted for print. The boxes in (B) indicate two critical regions of interest.

as well as reduce the number of pixels under consideration, pixels which are not along the center-lines of edge features must be damped or eliminated entirely. Anisotropic nonmaxima suppression using feature orientation estimates, as employed by Canny [32], is a well-known method using the insight that a ridge point ought to occur at a peak in the projection along the direction normal to the ridge. On the other hand, orientation estimates may be subject to noise, and the exact pixel location of a numerical maximum may deviate from the perceptual edge, leading to broken ridge-lines. Although the phase angles computed as intermediates in the phase congruency procedure may be used as feature orientation estimates for nonmaxima suppression, we take a different approach to ridge detection, one which does not rely on any such estimates.

Ridge detection is related to "thinning" of image features, in that the ridge is defined as the high-intensity center of a wider perceptual structure. The operation is therefore analogous to the morphological thinning of binary images. Unlike fundamental morphological operations such as erosion and dilation, the hit-or-miss transform from which homotopic binary thinning algorithms are derived is not well defined for intensity images, and to date grayscale thinning methods which are guaranteed to preserve image topology have proved elusive (see [37] for a review). The method in [38] is representative of a popular approach to grayscale thinning employing a connectivity heuristic which attempts to determine whether or not a pixel may be eroded. These connectivity-based methods can misidentify ridge points and display significant anisotropy, especially under the presence of noise.

Nevertheless, any binary image operation can be extended to grayscale by forming a linear superposition of the operation after global thresholding at all intensity values [39]. The intensities of a grayscale image are first quantized by restricting them to $N$ bins. The center of each bin is used as a global threshold to convert the quantized image to binary. A morphological operation is performed on the thresholded images, which are then summed to yield a new image under the same quantization. This technique is practically limited to relatively small bit-depths, due to the exponential proliferation of possible thresholds with increasing bit-depth, and down-sampling may

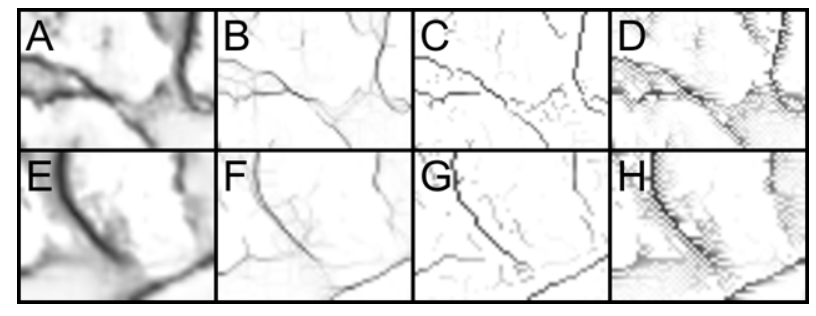

Fig. 8. Grayscale thinning by threshold superposition versus non-maxima suppression and connectivity-based thinning. (A)-(D) Phase congruency, grayscale thinning by threshold superposition, nonmaxima suppression and the connectivity-based thinning from [38], respectively, for ROI 1 in Fig. 7(b). (E)-(H) Same sequence, for ROI 2 in Fig. 7(b). All parts (A)-(H) inverted for print. Note that for both ROI only thinning by threshold superposition produces a thin, complete edge contour suitable for separating the parasites in Fig. 7(a).

be needed in order to reach useful levels of performance on available hardware.

Threshold superposition can be used to take advantage of the relationship between ridge detection and skeletonization [40]. In our work, grayscale thinning by threshold superposition is used to thin the phase congruency edge weights. A mathematical formulation of threshold superposition requires the global threshold operator $T_{t}$ defined over a set of pixels $X=\left\{x_{i}\right\}$ with intensities $I_{x}$ as given in

$$
T_{t}=\left\{x_{i} \in X \mid I\left(x_{i}\right)>t\right\} .
$$

Grayscale thinning by superposition is given by (10). The notation thin $_{\infty}$ indicates an infinite (binary) morphological thinning operation. Note that the phase congruency $P C_{i}$ is normalized by definition

$$
W_{i}=\sum_{t=0}^{N-1} \operatorname{thin}_{\infty}\left(T_{t}\left(\operatorname{round}\left(P C_{i} \cdot N\right)\right)\right) .
$$

The parallel thinning algorithm of Guo and Hall [41], which is simple to implement and obtains good results, is applied until convergence at each threshold independently. In practice, the procedure is optimized by restricting $t$ to values present in the image. As shown by Figs. 7 and 8, thinning by threshold superposition proves significantly more robust than both nonmaxima suppression and the connectivity-based thinning from [38], which leave gaps along the edge contour. The connectivity-based thinning result is also not completely thin. The strength of the threshold superposition approach derives from its use of a true topology-preserving thinning which ensures that the homotopic content of each possible horizontal cross section of the intensity surface is maintained. Thus, a ridge is localized by its common barycenter across all intensities, allowing detection of weak edge points and leading to complete edge contours.

Once the thinned edge weights are available, a binary edge image is determined using hysteresis thresholding. Hysteresis consists in locating pixels which are above a high threshold, or which are above a low threshold and are 8-connected to a pixel above the high threshold. In terms of binary sub-images given by high and low global threshold operations $T_{H}$ and $T_{L}$, the hysteresis edges may be written as the components of $T_{L}$ which are supersets of $T_{H}$ 


$$
E_{i}=\prod_{i} T_{L}\left(W_{i}\right) \supseteq \prod_{i} T_{H}\left(W_{i}\right) .
$$

The high threshold is taken to be that determined for the (thinned) PC image using Otsu's method; the low is taken as that value times $1 / 4$;. The hysteresis thresholds are thus reflective of the intensity distribution within each sub-image. The product of hysteresis is further processed by another round of binary morphological operations: bridging, thinning and isolated pixel removal [Fig. 3(g)]. These operations ensure that the edges are one pixel wide and serve to reduce the number of pixels which must be considered in the subsequent section.

\section{Topological Correction by Maximum Parsimony}

Binary edge set in hand, we first eliminate edges which cannot possibly create new regions. These irrelevant edges are located and eliminated as follows. A marker image is generated by direct subtraction of the edge set from the initial segmentation [Fig. 3(h)]. A given edge pixel is considered relevant if and only if its 8-neighborhood contains more than one marker region. The labeling operation is denoted as label 8 and the set of unique elements about a pixel $x$ is denoted as unique $_{8}$

$$
\begin{aligned}
M_{i} & =\operatorname{label}_{8}\left(C_{i}-E_{i}\right) \\
F_{i} & =\left\{x \in M_{i} \| \text { unique }_{8}(x) \mid>1\right\} .
\end{aligned}
$$

The relevant edges $F_{i}$ [Fig. 3(i)] constitute one pixel wide, 8 -connected edge contours, which nearly always determine the correct region connectivity, but may have rough or incorrectly placed boundaries due to noise structure in the image. Regions are split using a set of pixels derived from the relevant edges which satisfies the principle of maximum parsimony in that it is the minimum set required to obtain the correct topology from the initial segmentation. This set is equivalent to the watershed lines of the outward distance transform of the corrected binary image - pixels which will always be reached last by the priority queue used for flooding calculations. This differs somewhat from the standard use of watershed transformation for image segmentation in that only the pixels between objects that have already been separated are considered and assigned to an object. This task was originally performed by an infinite morphological thickening, but the watershed transform proved to be more robust and significantly faster to compute. It should be noted that the watershed lines are not necessarily subsets of the relevant edges $F_{i}$ from which they are derived. Using the watershed transform to identity the minimum edge set that obtains the desired connectivity, the corrected sub-images $D_{i}$ [Fig. $3(\mathrm{~m})$ and (n)] are given by (14). $\mathcal{S}(X)$ denotes the watershed lines of the distance transform of a binary image $X$

$$
D_{i}=C_{i}-\mathcal{S}\left(C_{i}-F_{i}\right) .
$$

\section{Cleanup and Reassembly}

In the final step, the corrected sub-images are reassembled into the final segmentation result. Each sub-image is added to the output $R_{f}$ by repeated union

$$
R_{f}=\bigcup_{i} D_{i}
$$

As the processing is carried out on individual connected components from the initial segmentation, there is no danger of the repeated union introducing a new merge or affecting the Euler number in any other way. Once the reassembly is complete, any remaining holes are filled and regions which overlap the image border are cleared. Due the selective erosion described above, parasites originally in limited contact with the border may be severed from the border so that the information they represent is not removed in this terminal step.

\section{PARAmeter SELECTION}

Of the parameters employed by the proposed method, only the global threshold $\gamma$ used in (1) to find the initial foreground mask is critical. This parameter is estimated programmatically (see Section IV). The robustness of the maximum likelihood estimation procedure is demonstrated by Table I, which lists precision and recall values for the methods described in Section IV. Together with Fig. 4, these data attest to the criticality of the thresholding parameter.

The majority of the other parameters pertain to the relative scale of the parasites, which are about 100 pixels on a side, within a microscope image of $1388 \times 1040$ pixels. Such scale parameters include the size of the structuring element used for lighting correction, the scale of the Gaussian low-pass filter during initial segmentation, and the number and center wavelengths of the log-Gabor filters used for edge detection. None of these parameters are sensitive, and can be simply estimated so that the possible size range of parasites and their perceptual features are covered. The scale factor of the RBDF is 2 (giving a $90 \%$ contour with a diameter of about 10 pixels). Most parasite edges then have a width of one or two standard deviations in terms of the Gaussian low-pass filter. Initial segmentation also involves a selective erosion process described above. The structuring element of the general erosion is sized to match the low-pass filter, while an object contacting the border must do so with only 10 pixels if it is to be preserved.

Other scale parameters are used for phase congruency; during edge detection, full coverage of the range of scales exhibited by perceptual features of parasites is attained using five wavelet filters with center wavelengths ranging from 3 to 58 pixels. The ratio of the standard deviation of the log-Gabor filter in frequency space to the center frequency of each filter must remain the same. This parameter $\kappa / \omega_{0}$ is set so the bandwidth of each filter is sufficient so as to obtain good coverage of the entire frequency spectrum, which can be checked by examining the sum of the filters themselves. The stability of the phase congruency image with respect to these primary scale parameters is demonstrated by the lack of significant differences amongst the parts of Fig. 9. Each of these parts is calculated using each of four filter sizes and wavelength ranges, specified by $\kappa / \omega_{0}$ and the minimum wavelength $\omega_{\min }$. When $\omega_{\min }=5$, the maximum wavelength $\omega_{\max }=97.24$.

A final group of parameters does not pertain to scale. These include the number of discrete values $N$ used for threshold su- 


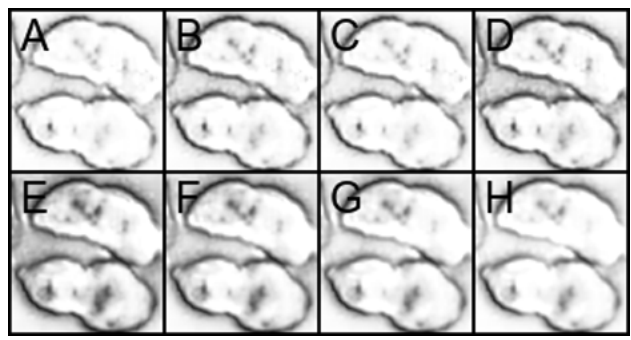

Fig. 9. Phase congruency with different parameter choices. (A) $\omega_{\min }=3$. $\kappa / \omega_{0}=0.4$. (B) $\omega_{\min }=3, \kappa / \omega_{0}=0.45$. (C) $\omega_{\min }=3, \kappa / \omega_{0}=0.5$. (D) $\omega_{\min }=3, \kappa / \omega_{0}=0.55$. (E) $\omega_{\min }=5, \kappa / \omega_{0}=0.4$. (F) $\omega_{\min }=5$, $\kappa / \omega_{0}=0.45$. (H) $\omega_{\min }=5, \kappa / \omega_{0}=0.5$. (A) $\omega_{\min }=5, \kappa / \omega_{0}=0.55$.

TABLE II

PARAMETER VALUES

\begin{tabular}{ll}
\hline Global threshold, $\gamma$ & Data driven \\
Tophat filter structuring element & 200 pixels square \\
RBDF scale, $a$ & 2 \\
Erosion size & 3 pixels square \\
Border overlap limit & 10 pixels \\
Wavelet scales & 5 \\
$\omega_{0}$ (wavelet center frequency) & $3,6.3,13.23,27.78,58.34$ \\
$\kappa / \omega_{0}$ (effective filter deviation) & 0.40 \\
$\beta$ (weight harshness) & 10 \\
$c$ (weighting cutoff) & 0.5 \\
$N$ (number of thresholds) & 255 \\
Minimum object size & 500 pixels \\
\hline
\end{tabular}

perposition as well as the sharpness $\beta$ and cutoff $c$ of the frequency spread weighting function. The respective values of 10 and 0.5 for these parameters are taken from [34] and are generally suitable. All of the parameters and their values are listed in Table II.

\section{EXPERIMENTS}

The algorithm we have described is designed to satisfy the criteria for accuracy laid out in Section I-C, and to mitigate the specific challenges presented by data from HTS of Schistosoma. As discussed previously, it is especially important that accuracy be maintained across the diverse phenotypes exhibited by individual schistosomes, even when this diversity is exacerbated by drug insult. It is therefore necessary to evaluate segmentation results under a variety of experimental conditions, including control conditions as well as extreme phenotypes which occur due to the action of different compounds. We draw from the diverse drug classes of the statins, protease inhibitors and anti-psychotics, all targeting human proteins likely to have analogues in schistosoma. In particular, phenotypes occurring due to the action of nine drugs-Acepromazine (Ace), Alimemazine (Ali), Chlorophenothiazine (2CPT), K11777, Pravastatin (Pra), PZQ, Promazine (Pro), Rosuvastatin (Ros), and Simvastatin (Sim) - are chosen as highly divergent parasite responses. In Section VIII we examine the quantitative and qualitative accuracy of the proposed method when applied to images of parasites exposed to these molecules (plus controls), as well as its suitability for high-throughput screening. In addition to the proposed method, we analyze the performance of Active Mask, watershed segmentation using Otsu's method, and watershed segmentation using ilastik (all described in Section II), which were deemed sufficiently promising to be subjected to a rigorous quantitative evaluation. A comparative evaluation is made between the proposed method, these three alternatives, as well as a variation on the proposed method which substitutes the Canny edge detector for that described in Section V-A.

\section{A. Quantitative Evaluation of Segmentation Accuracy}

Quantitative assessment of segmentation accuracy is made by comparing segmentation results to an extensive corpus of images carefully hand-segmented using a touchscreen computer. This library of ground truth data contains manual segmentations of eight different positions each of 870 unique parasites drawn from 200 images. The overall diversity of this set can be demonstrated by the following statistics: parasite areas range from 730 to 6911 pixels, perimeters from 105 to 442 pixels, the proportion of the bounding box filled from 0.22 to 0.81 and their mean intensities from 112 to 210 . These images (frames) are drawn from videos representing control conditions (five videos, 40 frames), as well as each of the compounds listed above ( 20 videos, 160 frames). The selected experiments all involved high concentrations $(1 \mu \mathrm{M})$ and varying exposure durations (1-7 days). As a result, the hand-segmented parasites embody a vast range of apparent and behavioral phenotypes. Furthermore, clustered, touching parasites in each frame were noted (1712 instances of 214 individuals). These clusters of touching parasites were used to closely examine segmentation accuracy in the most difficult cases, identified as an especial concern in Section I-C. Finally, two positions each of 198 parasites were independently hand-segmented by three volunteers, to determine the baseline variability of manual data analysis (i.e., the ability of each volunteer to predict the results of the others). Quantification of variation between human observers is found in Table IV. Naturally, the time taken for hand-segmentation is highly dependent on the number of parasites in an image. The minimum time taken for any image was $7 \mathrm{~min}$, with an average of about $16 \mathrm{~min}$, underlining the need for effective, automated segmentation.

The ground truth data was used to assess segmentation accuracy in terms of four measures.

1) Pixel-by-pixel precision of segmentation (probability that a segmented pixel is from the foreground).

2) The pixel-by-pixel recall of segmentation (probability that a foreground pixel is segmented).

3) Object-count agreement.

4) Average deviation of object boundaries.

Object-count agreement (OCA) is defined as the fractional object count discrepancy $O=\left(\left|N_{S}-N_{0}\right| / N_{0}\right)$ where $O$ is the discrepancy, $N_{0}$ the true number of objects and $N_{S}$ the number of objects in a given binary mask.

Deviation between boundaries is estimated by calculating the Euclidean distance transform of the ground truth object boundaries and finding the mean value of the points found on the boundaries of the objects from the trial segmentation. This represents the mean boundary deviation (MBD). Each metric is taken as an average across multiple images, weighted by the number of objects in each image. 
TABLE III

DETAILED QuantiTATIVE EVALUATION

\begin{tabular}{|c|c|c|c|c|c|c|c|c|c|c|}
\hline \multirow[b]{2}{*}{ Drug } & \multicolumn{5}{|c|}{ Precision } & \multicolumn{5}{|c|}{ Recall } \\
\hline & Proposed & $\begin{array}{c}\text { Proposed/ } \\
\text { Canny }\end{array}$ & $\begin{array}{c}\text { Active } \\
\text { mask }\end{array}$ & $\begin{array}{c}\text { Otsu/ } \\
\text { Watershed }\end{array}$ & $\begin{array}{c}\text { llastik/ } \\
\text { Watershed }\end{array}$ & Proposed & $\begin{array}{c}\text { Proposed/ } \\
\text { Canny }\end{array}$ & $\begin{array}{l}\text { Active } \\
\text { mask }\end{array}$ & $\begin{array}{c}\text { Otsu/ } \\
\text { Watershed }\end{array}$ & $\begin{array}{c}\text { Ilastik/ } \\
\text { Watershed }\end{array}$ \\
\hline Ace & 0.975 & 0.984 & 0.953 & 0.985 & 0.873 & 0.919 & 0.927 & 0.704 & 0.869 & 0.974 \\
\hline Ali & 0.952 & 0.968 & 0.991 & 0.586 & 0.925 & 0.949 & 0.926 & 0.690 & 0.711 & 0.953 \\
\hline $2 \mathrm{CPT}$ & 0.984 & 0.983 & 0.973 & 0.755 & 0.875 & 0.944 & 0.945 & 0.694 & 0.825 & 0.975 \\
\hline K11777 & 0.961 & 0.969 & 0.983 & 0.966 & 0.927 & 0.955 & 0.944 & 0.682 & 0.825 & 0.963 \\
\hline Pra & 0.927 & 0.959 & 0.954 & 0.302 & 0.898 & 0.973 & 0.939 & 0.770 & 0.600 & 0.971 \\
\hline Pro & 0.975 & 0.987 & 0.938 & 0.935 & 0.866 & 0.951 & 0.920 & 0.723 & 0.795 & 0.973 \\
\hline PZQ & 0.935 & 0.950 & 0.978 & 0.908 & 0.910 & 0.937 & 0.932 & 0.614 & 0.730 & 0.968 \\
\hline Ros & 0.940 & 0.942 & 0.950 & 0.677 & 0.909 & 0.957 & 0.951 & 0.735 & 0.849 & 0.968 \\
\hline Sim & 0.900 & 0.935 & 0.983 & 0.953 & 0.918 & 0.978 & 0.960 & 0.752 & 0.777 & 0.969 \\
\hline Control & 0.964 & 0.980 & 0.987 & 0.866 & 0.902 & 0.928 & 0.923 & 0.673 & 0.835 & 0.969 \\
\hline Clustered & 0.959 & 0.972 & 0.975 & 0.830 & 0.898 & 0.933 & 0.915 & 0.670 & 0.772 & 0.971 \\
\hline \multirow[t]{2}{*}{ Average } & 0.953 & 0.967 & 0.973 & 0.821 & 0.902 & 0.946 & 0.935 & 0.693 & 0.789 & 0.968 \\
\hline & \multicolumn{5}{|c|}{ Object count discrepancy } & \multicolumn{5}{|c|}{ Mean boundary deviation } \\
\hline- & Proposed & $\begin{array}{c}\text { Proposed/ } \\
\text { Canny }\end{array}$ & $\begin{array}{l}\text { Active } \\
\text { mask }\end{array}$ & $\begin{array}{c}\text { Otsu/ } \\
\text { Watershed }\end{array}$ & $\begin{array}{c}\text { Ilastik/ } \\
\text { Watershed }\end{array}$ & Proposed & $\begin{array}{c}\text { Proposed/ } \\
\text { Canny }\end{array}$ & $\begin{array}{l}\text { Active } \\
\text { mask }\end{array}$ & $\begin{array}{c}\text { Otsu/ } \\
\text { Watershed }\end{array}$ & $\begin{array}{c}\text { Ilastik/ } \\
\text { Watershed }\end{array}$ \\
\hline Ace & 0.035 & 0.055 & 0.129 & 0.065 & 0.259 & 1.16 & 0.98 & 4.88 & 1.88 & 1.55 \\
\hline Ali & 0.050 & 0.080 & 0.075 & 0.552 & 0.130 & 1.29 & 2.20 & 6.47 & 12.66 & 5.47 \\
\hline $2 \mathrm{CPT}$ & 0.026 & 0.035 & 0.139 & 0.292 & 0.420 & 0.88 & 0.79 & 5.07 & 4.09 & 2.35 \\
\hline K11777 & 0.036 & 0.051 & 0.039 & 0.268 & 0.163 & 1.55 & 3.84 & 7.86 & 9.83 & 3.87 \\
\hline Pra & 0.098 & 0.100 & 0.117 & 0.984 & 0.081 & 0.97 & 1.03 & 3.82 & 20.95 & 2.47 \\
\hline Pro & 0.036 & 0.046 & 0.086 & 0.813 & 0.481 & 0.76 & 1.30 & 4.33 & 11.79 & 1.60 \\
\hline PZQ & 0.042 & 0.100 & 0.145 & 0.431 & 0.083 & 1.17 & 1.18 & 6.98 & 11.49 & 1.24 \\
\hline Ros & 0.049 & 0.094 & 0.098 & 0.299 & 0.129 & 1.67 & 1.68 & 5.54 & 6.03 & 3.43 \\
\hline Sim & 0.034 & 0.166 & 0.238 & 0.392 & 0.145 & 2.20 & 3.17 & 4.98 & 8.90 & 2.73 \\
\hline Control & 0.028 & 0.058 & 0.135 & 0.301 & 0.160 & 1.44 & 1.61 & 5.48 & 6.37 & 2.64 \\
\hline Clustured & 0.126 & 0.314 & 0.321 & 0.252 & 0.070 & 1.27 & 1.60 & 6.97 & 3.57 & 1.34 \\
\hline Average & 0.041 & 0.074 & 0.117 & 0.407 & 0.189 & 1.32 & 1.85 & 5.78 & 9.16 & 2.68 \\
\hline
\end{tabular}

Clustered parasites (second from bottom) represent all conditions. Precision and recall reflect general foreground accuracy, while object count discrepancy and boundary deviation reflect proper identification of individual parasites and placement of their boundaries, respectively. All values are means weighted by the number of parasites in each image. Results for the proposed method are shown in italics, and the best performance for each line in bold.

TABLE IV

ReProducibiLity of MANUAL SEgMENTATION

\begin{tabular}{ccccc}
\hline Condition & Precision & Recall & $\begin{array}{c}\text { Object count } \\
\text { discrepancy }\end{array}$ & $\begin{array}{c}\text { Mean } \\
\text { boundary } \\
\text { deviation }\end{array}$ \\
Drug exposed & 0.927 & 0.904 & 0.072 & 3.55 \\
Control & 0.957 & 0.948 & 0.041 & 2.89 \\
Clustered & 0.948 & 0.911 & 0.107 & 2.22 \\
Average & 0.935 & 0.915 & 0.065 & 3.39 \\
\hline
\end{tabular}

The results of these tests as applied to the proposed and alternative methods are summarized in Table III. The proposed method is shown to be quite accurate over a wide range of experimental conditions. In terms of precision and recall, which reflect accuracy of foreground segmentation but not separation of individual objects, the proposed method performs similarly to human experts (Table IV). Where the human values are exceeded, the algorithm is able to deliver a better estimate of the perceptual consensus than individual people. While some of the alternatives have similar performance, the proposed method is best when both precision and recall are taken into account.

Because boundary pixels comprise in general a small proportion of the foreground, it is necessary to employ OCA and MBD in order to determine accuracy of individual object recognition as well as placement of region boundaries, respectively. The MBD is particularly strict as any displacement of the boundary will have a large impact on the final value. The results for OCA and MBD indicate the proposed method has the ability both to recognize $96 \%$ individual parasites and to spatially locate their edges within 1.3 pixels on average. The relative consistency of these results across several extreme, drug-induced phenotypes is especially notable. In comparison, except for the variant of the proposed method, none of the alternatives recognize more than $90 \%$ of individuals or place boundaries more accurately than 2.7 pixels.

\section{B. Computational Complexity and Segmentation Speed}

We next address the suitability of the proposed method, as well as the reviewed alternatives, to the high-throughput scenario. At a high level, the complexity of the proposed method is linear in the number of objects, log-linear in the number of pixels in each object (due to FFT-based wavelet transform) and linear in the total size of the image (due to threshold estimation and watershed calculation). Table $\mathrm{V}$ lists real-world timing information for each method, based on a single thread running on a $2.6 \mathrm{GHz}$ AMD Athlon II processor ( 8 GB memory). Both the proposed method and Active Mask are implemented in MATLAB. The watershed segmentation variants were implemented using CellProfiler. Thus, these two methods indicate the power and limitations of the algorithms currently available within that framework. As Table V (and Table III) show, the proposed method strikes the best balance between segmentation quality and computational resources required; although the simpler variant using the Canny edge detector is somewhat faster, it is considerably less accurate. Each of the other methods is prohibitively slow for high-throughput 
TABLE V

SEGMENTATION SPEED

\begin{tabular}{cc}
\hline Algorithm & Time per frame (s) \\
Active Mask $^{\mathrm{a}}$ & $411.75^{\mathrm{c}}$ \\
Otsu / Watershed $^{\mathrm{b}}$ & 72.02 \\
Ilastik / Watershed $^{\mathrm{b}}$ & 110.32 \\
${\text { Proposed method } / \text { Canny }^{\mathrm{a}}}^{\mathrm{a}}$ & 2.87 \\
Proposed method $^{\mathrm{a}}$ & 9.28 \\
\hline${ }^{\mathrm{a}}$ Implemented in MATLAB. \\
${ }^{\mathrm{b} I m p l e m e n t e d ~ u s i n g ~ C e l l P r o f i l e r . ~}$ \\
${ }^{\mathrm{c}}$ Image resolution was reduced by half to obtain a reasonable runtime.
\end{tabular}

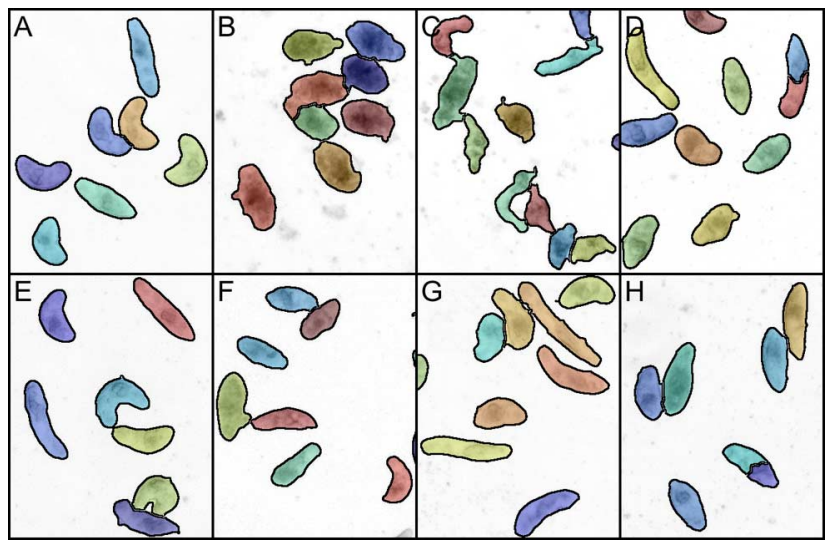

Fig. 10. Sample segmentation results under different conditions. (A) Control. (B) Sim. (C) PZQ. (D) K11777. (E) Pro. (F) Pra. (G) 2CPT. (H) Ali.

data analysis (processing $>10^{5}$ images), in addition to their reduced accuracies.

\section{Case Studies for Qualitative Evaluation}

Supplementing the above numerical analysis, Fig. 10 displays samples of segmentation using the proposed method. These case studies render additional examples of the challenges at hand, taken from representative control and drug-exposed parasites. As shown, under control conditions large groups of touching parasites are almost always correctly separated and the region boundaries are placed very close to their true locations. In comparison, long term exposure to some drugs causes the edges between touching parasites to become very weak. For this reason, parasites in these conditions are more difficult to separate when they are in physical contact and are not correctly segmented in all frames. Nevertheless, the segmentation is largely accurate and most touching parasites are still split into discrete regions, attesting to the sensitivity of phase congruency to perceptual edge features, as well as to the robustness of the method as a whole against the prevalence of debris and other noise sources.

Many drugs give rise to unique phenotypes. For example, the presence of PZQ in particular [Fig. 10(c)] evokes a peculiar phenotype in which the parasites tend to "shrivel," adopting very irregular shapes which often bear narrow protrusions from the body. Here, changes in the appearance of anatomical features within the parasites contribute to false edges with the potential to induce false splitting of single parasites. Despite this, greater than $95 \%$ of parasites are identified, and their boundaries are placed within 1.2 pixels of the location chosen by a human observer.

\section{CONCLUSION}

Neglected diseases such as schistosomiasis represent a serious global health issue. We have presented a segmentation algorithm designed specifically towards the segmentation of standard, bright-field microscopy images of schistosomes, which is robust against variegated natural and drug induced phenotypes, and which does not depend on any proprietary HTS systems.

The method includes a novel, high-sensitivity edge operator which, to the best of our knowledge, is the first to combine phase congruency with ridge-detection by grayscale thinning. We submit that due to the power and generality of the phase congruency approach to edge strength, and the highly conservative nature of grayscale morphological thinning by superposition, our detector has broad applicability in the relatively common case that there are edges at a wide range of phase angles, which may or may not have strong directional maxima across their entire contour.

Quantitative and qualitative analysis of segmentation results under multiple experimental conditions demonstrate the wide applicability of the algorithm. In the context of high-content screening, the use of the proposed method allows highly accurate measurement of visual and behavioral time series data for every individual parasite observed during an experiment. Such highly granular data permit holistic analyses of the disease system, and increase the likelihood of discovering drugs which may not result in death, but which nevertheless induce phenotypic changes that enable the immune system to fight back effectively or eliminates the disease outright.

It is hoped that the proposed method will underlie a truly high-throughput, phenotypic screen against schistosomiasis and lead both to the discovery of new drugs against this disease and lay ground work towards the application of HTS methods to other parasitic illnesses. We are currently engaged with our collaborators in producing a publically available repository for data from HCS of parasites, and in developing a publicly available software encompassing multiple stages of HCS data analysis, from segmentation to automated discovery of drug induced phenotypes.

\section{ACKNOWLEDGMENT}

The authors would like to thank C. Caffrey and B. Suzuki for video-recordings of the parasites, as well as for providing insight into the parasitology. The authors would also like to thank K. Finnegan, C. Bastian, and J. Cheasty for hand segmenting images.

\section{REFERENCES}

[1] D. W. T. Crompton, D. Daumerie, P. Peters, and L. Savioli, Working to overcome the global impact of neglected tropical diseases first WHO report on neglected tropical diseases. Geneva, Switzerland: World Health Org., 2010.

[2] C. R. Caffrey, "Chemotherapy of schistosomiasis: Present and future," Curr. Opin. Chem. Biol., vol. 11, no. 4, pp. 433-439, Aug. 2007.

[3] S. Nwaka and A. Hudson, "Innovative lead discovery strategies for tropical diseases," Nature Rev. Drug Discov., vol. 5, no. 11, pp. 941-955, Nov. 2006. 
[4] D. C. Swinney and J. Anthony, "How were new medicines discovered?," Nature Rev. Drug Discov., vol. 10, no. 7, pp. 507-519, Jun. 2011.

[5] R. Singh, M. Pittas, I. Heskia, F. Xu, J. McKerrow, and C. R. Caffrey, "Automated image-based phenotypic screening for high-throughput drug discovery," in Proc. 22nd IEEE Int. Symp. Comput.-Based Med. Syst. (CBMS 2009), 2009, pp. 1-8.

[6] H. Lee, A. Moody-Davis, U. Saha, B. M. Suzuki, D. Asarnow, S. Chen, M. Arkin, C. R. Caffrey, and R. Singh, "Quantification and clustering of phenotypic screening data using time-series analysis for chemotherapy of schistosomiasis," BMC Genomics, vol. 13, pp. S4-S4, Jan. 2012.

[7] R. A. Paveley, N. R. Mansour, I. Hallyburton, L. S. Bleicher, A. E. Benn, I. Mikic, A. Guidi, I. H. Gilbert, A. L. Hopkins, and Q. D. Bickle, "Whole organism high-content screening by label-free, image-based Bayesian classification for parasitic diseases," PLoS Neglected Tropical Diseases, vol. 6, no. 7, pp. e1762-e1762, Jul. 2012.

[8] K.-M. Huang, P. Cosman, and W. Schafer, "Using articulated models for tracking multiple C. Elegans in physical contact," J. Signal Process. Syst., vol. 55, no. 1, pp. 113-126, 2009.

[9] C. Wahlby, T. Riklin-Raviv, V. Ljosa, A. L. Conery, P. Golland, F. M. Ausubel, and A. E. Carpenter, "Resolving clustered worms via probabilistic shape models," in Proc. 2010 IEEE Int. Symp. Biomed. Imag.. From Nano to Macro, 2010, pp. 552-555.

[10] X. Bai, C. Sun, and F. Zhou, "Splitting touching cells based on concave points and ellipse fitting," Pattern Recognit., vol. 42, no. 11, pp. 2434-2446, Nov. 2009.

[11] G. Lin, M. K. Chawla, K. Olson, C. A. Barnes, J. F. Guzowski, C. Bjornsson, W. Shain, and B. Roysam, "A multi-model approach to simultaneous segmentation and classification of heterogeneous populations of cell nuclei in 3D confocal microscope images," Cytometry Part $A$, vol. 71A, no. 9, pp. 724-736, Sep. 2007.

[12] X. Chen, X. Zhou, and S. T. C. Wong, "Automated segmentation, classification, and tracking of cancer cell nuclei in time-lapse microscopy," IEEE Trans. Biomed. Eng., vol. 53, no. 4, pp. 762-766, Apr. 2006.

[13] P. S. U. Adiga and B. B. Chaudhuri, "An efficient method based on watershed and rule-based merging for segmentation of 3-D histo-pathological images," Pattern Recognit., vol. 34, no. 7, pp. 1449-1458, 2001.

[14] X. Yang, H. Li, and X. Zhou, "Nuclei segmentation using marker-controlled watershed, tracking using mean-shift, and Kalman filter in timelapse microscopy," IEEE Trans. Circuits Syst. I, Reg. Papers, vol. 53, no. 11, pp. 2405-2414, Nov. 2006.

[15] K. Z. Mao, P. Zhao, and P.-H. Tan, "Supervised learning-based cell image segmentation for P53 immunohistochemistry," IEEE Trans. Biomed. Eng., vol. 53, no. 6, pp. 1153-1163, Jun. 2006.

[16] C. Zimmer, E. Labruyere, V. Meas-Yedid, N. Guillen, and J.-C. Olivo-Marin, "Segmentation and tracking of migrating cells in videomicroscopy with parametric active contours: A tool for cell-based drug testing," IEEE Trans. Med. Imag., vol. 21, no. 10, pp. 1212-1221, Oct. 2002

[17] T. F. Chan and L. A. Vese, "Active contours without edges," IEEE Trans. Image Process., vol. 10, no. 2, pp. 266-277, Feb. 2001.

[18] W. Yu, H. K. Lee, S. Hariharan, W. Bu, and S. Ahmed, "Quantitative neurite outgrowth measurement based on image segmentation with topological dependence," Cytometry Part A, vol. 75A, no. 4, pp. 289-297, Apr. 2009.

[19] X.-C. Tai, E. Hodneland, J. Weickert, N. Bukoreshtliev, A. Lundervold, and H.-H. Gerdes, "Level set methods for watershed image segmentation," in Scale Space and Variational Methods in Computer Vision, F. Sgallari, A. Murli, and N. Paragios, Eds. Berlin, Germany: Springer, 2007, vol. 4485, pp. 178-190.

[20] P. Yan, X. Zhou, M. Shah, and S. T. C. Wong, "Automatic segmentation of high-throughput RNAi fluorescent cellular images," IEEE Trans. Inf. Technol. Biomed., vol. 12, no. 1, pp. 109-117, Jan. 2008.
[21] Y. Al-Kofahi, W. Lassoued, W. Lee, and B. Roysam, "Improved automatic detection and segmentation of cell nuclei in histopathology images," IEEE Trans. Biomed. Eng., vol. 57, no. 4, pp. 841-852, Apr. 2010 .

[22] J. Shi and J. Malik, "Normalized cuts and image segmentation," IEEE Trans. Pattern Anal. Mach. Intell., vol. 22, no. 8, pp. 888-905, Aug. 2000.

[23] C. Sommer, C. Straehle, U. Koethe, and F. A. Hamprecht, "ilastik: Interactive learning and segmentation toolkit," in Proc. 8th IEEE Int. Symp. Biomed. Imag., 2011, pp. 230-233.

[24] G. Srinivasa, M. C. Fickus, Y. Guo, A. D. Linstedt, and J. Kovacevic, "Active mask segmentation of fluorescence microscope images," IEEE Trans. Image Process., vol. 18, no. 8, pp. 1817-1829, Aug. 2009.

[25] A. E. Carpenter, T. R. Jones, M. R. Lamprecht, C. Clarke, I. H. Kang, O. Friman, D. A. Guertin, J. H. Chang, R. A. Lindquist, J. Moffat, P. Golland, and D. M. Sabatini, "CellProfiler: Image analysis software for identifying and quantifying cell phenotypes," Genome Biol., vol. 7, no. 10, pp. R100-R100, Oct. 2006.

[26] G. Srinivasa, M. C. Fickus, Y. Guo, A. D. Linstedt, and J. Kovacevic, "Active mask segmentation of fluorescence microscope images," IEEE Trans. Image Process., vol. 18, no. 8, pp. 1817-1829, Aug. 2009.

[27] A. Moody-Davis, L. Mennillo, and R. Singh, "Region-based segmentation of parasites for high-throughput screening," in Proc. 7th Int. Conf. Adv. Vis. Comput., Berlin, Germany, 2011, pp. 43-53.

[28] N. Otsu, "A threshold selection method from gray-level histograms," IEEE Trans. Syst., Man Cybern., vol. 9, no. 1, pp. 62-66, Jan. 1979.

[29] C. A. Glasbey, "An analysis of histogram-based thresholding algorithms," CVGIP: Graph. Models Image Process., vol. 55, no. 6, pp. 532-537, Nov. 1993.

[30] C. F. J. Wu, "On the convergence properties of the EM algorithm," Ann. Statist., vol. 11, no. 1, pp. 95-103, Mar. 1983.

[31] N. R. Pal and D. Bhandari, "Image thresholding: Some new techniques," Signal Process., vol. 33, no. 2, pp. 139-158, Aug. 1993.

[32] J. Canny, "A computational approach to edge detection," IEEE Trans. Pattern Anal. Mach. Intell., vol. 8, no. 6, pp. 679-698, Nov. 1986.

[33] M. C. Morrone and R. A. Owens, "Feature detection from local energy," Pattern Recognit. Lett., vol. 6, no. 5, pp. 303-313, Dec. 1987.

[34] P. Kovesi, "Image features from phase congruency," Videre, vol. 1, no. 3, pp. 1-26, 1999.

[35] H. Farid and E. P. Simoncelli, "Differentiation of discrete multidimensional signals," IEEE Trans. Image Process., vol. 13, no. 4, pp. 496-508, Apr. 2004.

[36] D. J. Field, "Relations between the statistics of natural images and the response properties of cortical cells," J. Opt. Soc. Am. A, vol. 4, no. 12 pp. 2379-2394, Dec. 1987.

[37] K. Saeed, M. Tabedzki, M. Rybnik, and M. Adamski, "K3M: A universal algorithm for image skeletonization and a review of thinning techniques," Int. J. Appl. Math. Comput. Sci., vol. 20, no. 2, pp. 317-335, Jun. 2010.

[38] C. Wang and K. Abe, "A method for gray-scale image thinning: The case without region specification for thinning," in Proc. 11th IAPR Int. Conf. Pattern Recognit., Conference C: Image, Speech Signal Anal., 1992, vol. 3, pp. 404-407.

[39] P. Maragos and R. D. Ziff, "Threshold superposition in morphologica image analysis systems," IEEE Trans. Pattern Anal. Mach. Intell., vol. 12, no. 5, pp. 498-504, May 1990.

[40] J. Weiss, "Grayscale thinning," presented at the ISCA 17th Int. Conf. Comput. Appl., San Francisco, CA, 2002.

[41] Z. Guo and R. W. Hall, "Parallel thinning with two-subiteration algorithms," Commun. ACM, vol. 32, no. 3, pp. 359-373, Mar. 1989. 